\title{
Ascenso y ocaso de una marca: la literatura hispanoamericana en Holanda
}

\section{Borges y la literatura latinoamericana}

¿Quién es Jorge Luis Borges? Con estas palabras arranca el largo texto de la contracubierta de De Aleph ${ }^{1}$, el primer libro del escritor argentino traducido al holandés, una selección de los cuentos en Ficciones y El Aleph. De Aleph fue publicado en 1964 por De Bezige Bij, una de las editoriales literarias más importantes de los Paises Bajos. Se diría y esperaría que las palabras que siguen a esta pregunta introdujeran a un autor desconocido que requiere una identidad que posibilite pasar el umbral y llegar a una nueva entidad de lectores. Esta expectativa, sin embargo, no se cumple. El texto que sigue sostiene que Borges ya tenía cierta fama antes de que aparecieran las primeras traducciones en el Viejo Mundo:

En Europa occidental [Borges era] un gran nombre y nada más: era un gran misterio y no se lo leía. Por fin podemos comprobar en Holanda si es justificada esta fama, después de las traducciones de su obra publicadas en Francia, Alemania e Italia, donde se convirtió en uno de los grandes descubrimientos de la literatura de la posguerra.

La contextualización se continúa en el resto del texto que llena la contracubierta. Así, el siguiente párrafo caracteriza a Borges como un escritor argentino genial e ingenioso que maneja un estilo riguroso y, además, califica su obra como laberíntica, fantástica y muy imaginativa. El tercer párrafo es una pequeña biografía que destaca la estancia de Borges en Europa, la diversidad de su obra (poesía, cuentos, ensayos, críticas de cine, traducciones), su trabajo como director de la Biblioteca Nacional de Argentina y como catedrático de literatura inglesa y norteamericana en la Universidad de Buenos Aires. Luego, se menciona el Premio Formentor que el escritor argentino compartió en 1961 con Samuel Beckett y, después, se afirma que, según el semanario francés Arts, Borges es uno de los escritores más importantes del mundo y que Le Figaro lo ha calificado como uno de los grandes maestros de la literatura.

De un lado, queda claro que esta contextualización de Borges por De Bezige Bij se singulariza por su volumen y diversidad: incluye aspectos geográficos, literarios,

1 Todas las traducciones de los paratextos son del autor de este artículo.

Maarten Steenmeijer, Radboud University Nijmegen

Ә Open Access. ( 2020 Maarten Steenmeijer, published by De Gruyter. (cc)BY-NC-ND This work is licensed under a Creative Commons Attribution-NonCommercial-NoDerivatives 4.0 International License.

https://doi.org/10.1515/9783110673678-004 
profesionales, institucionales y mediáticos. Del otro, llama la atención que brille por su ausencia la etiqueta "hispanoamericano" o variantes de la misma como "latinoamericano" o "suramericano”. No es difícil de explicar: a principios de los años sesenta la literatura hispanoamericana todavía no fue percibida como tal en Holanda. Es cierto que en años anteriores a la aparición de De Aleph se habían publicado traducciones de Los pasos perdidos (Alejo Carpentier), de Hombres de maíz (Miguel Ángel Asturias) y Pedro Páramo (Juan Rulfo), pero estas fueron lanzadas por distintas editoriales, ninguna de las cuales presentó a estos nuevos autores como un conjunto. Este patrón se consolidó en los años posteriores:

[E]n Holanda la nueva novela hispanoamericana todavía no existía en los años 60. Solo había traducciones dispersas que apenas acumularon capital económico y literario, y que no habían conseguido que la literatura hispanoamericana llegara a formar parte del repertorio o la memoria colectiva de los lectores profesionales, por no hablar de los lectores comunes y corrientes. (Steenmeijer 117)

Para resumir: la literatura hispanoamericana distaba mucho de ser una marca, es decir “[a] unique design, sign, symbol, words, or a combination of these, employed in creating an image that identifies a product and differentiates it from its competitors” (“[a] unique design”). Sí que hubo dos autores cuyas obras echaron raíces en el sistema literario holandés en el curso de los años sesenta, como atestigua el número de libros traducidos, su excelente y amplia recepción crítica y, asimismo, las reediciones de algunos de ellos: Jorge Luis Borges y Julio Cortázar. De este modo, los dos escritores argentinos no tardaron en convertirse en marcas pero -importa recalcarlo- no en el marco de la literatura latinoamericana sino como autores idiosincráticos o mundiales.

Es en los años setenta cuando la literatura latinoamericana se canoniza como una marca en el campo literario holandés gracias, ante todo, a los esfuerzos de la editorial Meulenhoff, que en poco tiempo publicó, entre otras, todas las obras narrativas de García Márquez y Vargas Llosa, y gran parte de la obra de Carpentier y Cortázar (y luego también de Fuentes). Mientras tanto, De Bezige Bij siguió lanzando nuevos títulos de Borges al mercado holandés: libros de cuentos pero también de ensayos y de poesía. De esta manera, se desarrolló y potenció la marca Borges que, como tal, llegó a representar "certain benefits and values” y a ser asociada "with a level of credibility, quality, and satisfaction in the consumer's mind (...)” (“[a] unique design”).

No sería exagerado afirmar que a lo largo de los años Borges se convirtió en un clásico en Holanda y, de esta manera, llegó a formar parte del repertorio que los críticos y los lectores manejan para contextualizar (la obra de) otros autores. 
Es por ende muy curioso e interesante el anuncio publicado en abril de 1977 por De Bezige Bij para promocionar la obra del escritor argentino. El anuncio incluye una lista de las obras de Borges publicadas por su editorial holandesa y, además, una foto y el nombre del autor. Sorprendentemente, el conjunto queda encabezado por un prominente titular que reza "Zuidamerikaan" (Suramericano), epíteto que en este contexto ejemplifica la potencia de la literatura suramericana (o latinoamericana o hispanoamericana) como marca en aquellos años.

\section{La literatura escrita por mujeres}

Con el lanzamiento de la versión holandesa de La casa de los espíritus en 1985 por la editorial Wereldbibliotheek se introdujo una nueva vertiente de la literatura hispanoamericana en Holanda: la literatura escrita por mujeres, notoriamente ausente en el boom de los años anteriores. El texto en la contracubierta de Het huis met de geesten -el título de la versión neerlandesa- tiene tres partes. La primera refiere al golpe de estado de 1973 en Chile, un acontecimiento histórico que en aquel entonces formaba parte de la memoria colectiva de Holanda:

Het huis met de geesten es una crónica familiar que abarca cuatro generaciones desde principios del siglo veinte hasta los años setenta, cuando la dictadura terminó con el gobierno socialista de Salvador Allende.

La segunda parte (la más larga) ofrece un adecuado resumen de la trama. En la tercera parte se caracteriza la novela en las siguientes palabras:

En Het huis met de geesten Isabel Allende retrata la sociedad suramericana en todas sus formas heterogéneas y, muchas veces, también místicas y fantásticas. Con este libro Isabel Allende muestra que no solo es una escritora excepcionalmente dotada sino también y sobre todo una narradora de pura cepa.

Llama la atención que los paratextos de Het huis met de geesten no hagan ninguna referencia explícita a la literatura latinoamericana y tampoco a los autores que en los años anteriores habían reunido mucho capital literario y no poco capital económico en el sistema literario holandés: ni en la cubierta (donde solo se mencionan el nombre de la editorial, el de la autora, el título y el género del libro) ni en la contracubierta ni en la minibiografía en la primera página del libro, en la que, dicho sea de paso, también se menciona el golpe militar de $1973 \mathrm{y}$ donde se añade que Salvador Allende es el hermano del padre de la autora. La estrategia es otra: se ubica Het huis met de geesten en un contexto histórico concreto y conocido, se menciona, casi sobre la marcha, el lazo familiar entre la 
autora y Salvador Allende, se presenta la novela como una fiel representación de "la sociedad suramericana" (una significativa generalización, por cierto) y se alaban las capacidades narrativas de la autora. Es decir, por un lado la editorial vincula a la escritora y su novela al continente que se había puesto de moda en los años anteriores pero, por el otro, Wereldbibliotheek elude cualquier asociación con los autores latinoamericanos ya establecidos, representando a Isabel Allende como una escritora literariamente soberana pero arraigada en un espacio histórico y cultural que les era familiar a los lectores holandeses.

La crítica literaria, sin embargo, no dejó de señalar importantes parentescos con Cien años de soledad, y ello casi siempre en detrimento de la novela de Allende. Tampoco dejó de destacar ciertos estereotipos. Pero esto no impidió que la estrategia de Wereldbibliotheek - no aludir a (o competir con) los valores consagrados masculinos de la literatura latinoamericana sino destacar las propias calidades de la obra de Allende- funcionara magníficamente. Isabel Allende encontró su propio lugar en el mercado holandés: ha vendido (y sigue vendiendo) muchísimos libros (Het huis met de geesten ya lleva cuarenta y ocho ediciones y cada uno de los numerosos libros posteriores le siguieron las huellas), tiene un gran aprecio entre sus numerosos lectores (lectoras, sobre todo) mientras que tampoco le falta cierto prestigio literario institucional ${ }^{2}$. En resumidas cuentas: la escritora chilena se convirtió en una marca que se iba constituyendo fuera del contexto de la literatura latinoamericana establecida, monopolizada por discursos masculinos. Lo hizo, además, en base a un contexto histórico-cultural que en un principio era predominantemente local (Latinoamérica), pero que luego se iría globalizando debido a la hibridación de sus temáticas y personajes en novelas como El plan infinito (1991), El amante japonés (2015) y Mas allá del invierno (2017), vertebrada esta última en torno a una chilena de origen croata que va a trabajar en Nueva York, una guatemalteca sin papeles que se busca la vida en los Estados Unidos y un judío neoyorquino de origen europeo que se casó con una brasileña.

Siguiendo el camino de Isabel Allende, en los años noventa del siglo pasado las obras de autoras como Laura Esquivel y Gioconda Belli entraron en el mercado holandés. La primera lo hizo de forma espectacular con la versión holandesa de Como agua para chocolate (lanzada en 1991 bajo el curioso título de

2 Así, en 2001 la escritora chilena dio la Van der Leeuw-lezing (Conferencia Van der Leeuw) en Groningen, compartiendo este honor con Barbara Tuchman, Amos Oz, Simon Schama, Hans Magnus Enzensberger, Carlos Fuentes, Ben Okri, Ian McEwan y muchos otros prominentes escritores y pensadores. 
Rode rozen en tortilla's, es decir "Rosas rojas y tortillas”). Así como los paratextos de Het huis met de geesten, los de Rode rozen en tortilla's no vinculan la novela a la narrativa latinoamericana. Las únicas referencias al subcontinente en la contracubierta son el dato de que Laura Esquivel nació en México y el nombre de la autora de la cita que forma parte de los paratextos en la contracubierta: Elena Poniatowska (aunque es más que probable que los lectores holandeses no la asocien con México por su apellido ya que Poniatowska es una autora desconocida en Holanda). A diferencia de los de Het huis met de geesten, los paratextos de Rode rozen en tortilla's no destacan las capacidades narrativas de la autora, restringiéndose a resaltar la temática culinaria, sentimental, erótica y mágica de la novela. La frase que domina la cubierta refuerza la idea de que se trata de una novela exótica y sentimental: "En Méjico una mujer le prepara platos tradicionales a su amor inalcanzable” (Esquivel).

Rode rozen en tortilla's fue, además de un best seller, un long seller: en 2017 apareció la trigésima tercera edición. Las novelas de Esquivel que luego se publicaron (traducciones de La ley del amor, Tan veloz como el deseo, Malinche), empero, ni por asomo lograron tener el éxito que tuvo Como agua para chocolate. Como consecuencia y a diferencia de Isabel Allende, la autora se quedó en la sombra de la novela con la que debutó. Pero lo que importa aquí es comprobar que, igual que en el caso de la escritora chilena, ninguna de las dos -autora y novela- fue vinculada a la marca establecida de la literatura latinoamericana. Lo mismo vale para Gioconda Belli, otra autora del subcontinente que en Holanda debutó como novelista a principios de los años noventa. La editorial De Geus lanzó De bewoonde vrouw (1992, La mujer habitada) como una novela arraigada en la cultura y la historia de Latinoamérica (dictadura; guerrillas; machismo; indios) sin referir al boom, a la nueva novela o alguno de los autores consagrados como Gabriel García Márquez, Mario Vargas Llosa o Carlos Fuentes. A ello es importante añadir que en Holanda, curiosa y significativamente, las editoriales no aprovecharon la oportunidad de crear una nueva marca: no se empeñaron en lanzar a autoras como Isabel Allende, Laura Esquivel y Gioconda Belli como una nueva tendencia de la literatura latinoamericana, es decir, la literatura escrita por mujeres. Con ello, el concepto "literatura latinoamericana” seguía siendo monopolizado por los viejos valores masculinos del boom de la nueva novela. Como tal, estaba en vías de perder rigor y vigor, como se detallará más abajo. 


\section{Eterno El Dorado}

Desde 1932 en Holanda se organiza cada año con bombo y platillo La Semana del Libro (De Boekenweek). En 1996 el tema del evento fue la literatura latinoamericana (y la caribeña, para incluir y resaltar las literaturas de Surinam y las Antillas Holandesas). La relevancia o urgencia del tema -lanzado bajo el lema de "Eterno El Dorado" - no era evidente para todos, como atestiguan las siguientes palabras del crítico literario Willem Kuipers vertidas en el largo artículo que escribió con motivo del evento en de Volkskrant, uno de los periódicos nacionales más importantes de Holanda:

¿Por qué? ¿Por qué era tan atractiva la literatura de Latinoamérica y del Caribe y por qué se acabó? ¿Por qué se organiza ahora esta revitalización? ¿Algo que estaba atrofiándose tiene que ser reanimado con una última transfusión de sangre?

Luego, Kuipers pasa revista a algunos de los libros que se publicarían en el marco de "Eterno El Dorado" y ensalza a autores como Jorge Luis Borges, Mario Vargas Llosa, Gabriel García Márquez, Julio Cortázar, Carlos Fuentes, Juan Carlos Onetti y Homero Aridjis (así como a algunos escritores brasileños, que quedan fuera del marco de este artículo). Llama la atención que no figure ninguna escritora entre los autores comentados por Kuipers. Es sintomática, asimismo, la ausencia de escritores más jóvenes como Luis Sepúlveda, el chileno que con tres novelas ya estaba bien representado en el prestigioso catálogo de Meulenhoff: Un viejo que leía novelas de amor (1993), Mundo del fin del mundo (1994) y Nombre de torero (1995). Con todo, tanto las presencias como las ausencias en el artículo de Kuipers tienden a constituir una respuesta afirmativa a las preguntas planteadas por el crítico: pues no, no tenía mucho sentido organizar la Semana del Libro en torno a una literatura que ya había alcanzado su cénit y estaba perdiendo importancia e interés.

Esta misma idea predomina en los comentarios de algunos expertos entrevistados sobre el tema de la Semana del Libro de 1996. Reinjan Mulder, crítico literario del diario nacional NRC Handelsblad, sostuvo que "En primer lugar, se trata de un tema que no corresponde con la actualidad literaria. En segundo lugar, los editores podrían haber buscado de forma más activa a nuevos autores. Los libros que ahora están promocionando son todos reediciones” (De Vries). Igual de categóricas son las palabras de la celebrada traductora Barber van de Pol: "La literatura latinoamericana no está en su mejor momento, recientemente no se ha descubierto ningún magnífico escritor. Hay interés, pero la euforia de hace quince años se ha debilitado" (De Vries). 


\section{4 ¿Un boom Bolaño?}

Roberto Bolaño (Santiago, Chile, 1953) es considerado como uno de los mayores talentos de la literatura latinoamericana. Publicó algunas novelas y un libro de cuentos, y fue galardonado con varios premios, entre ellos el Premio Rómulo Gallegos, que antes había sido otorgado a, entre otros, Gabriel García Márquez y Mario Vargas Llosa. (De woeste zoekers)

Esta minibiografía es el texto con el que la editorial Meulenhoff introdujo a Roberto Bolaño en la contracubierta de De wilde zoekers (Los detectives salvajes), el primer libro del escritor chileno traducido al holandés. Hay más paratextos en la contracubierta que revelan los esfuerzos de Meulenhoff de vincular a Bolaño a la gran narrativa latinoamericana que hacía unas décadas había tenido un gran impacto en Holanda. Así, figura una cita de El País que vincula Los detectives salvajes a Borges: "El tipo de novela que Borges hubiera aceptado escribir. . Un libro original y hermosísimo, divertido, conmovedor, importante” (De woeste zoekers). También vale la pena mencionar la cita del semanario alemán Der Spiegel que forma parte de los paratextos de la contracubierta de De wilde zoekers y que califica a Bolaño "como probablemente lo más original que la literatura escrita en español hoy en día nos ofrece”.

Estos paratextos vuelven en las contracubiertas de Het lichtende kwaad (la versión holandesa de Estrella distante que vio la luz en 2002) y de Chileense nocturne (Nocturno de Chile, 2004) aunque, igual que en el caso de De wilde zoekers, no surtieron el efecto deseado. No es que faltaran críticas positivas sobre estas tres novelas de Bolaño, pero eran pocas. Demasiado pocas como para conseguir que la obra de Bolaño echara raíces en Holanda. Otra suerte tuvo la versión holandesa de 2666, publicada en 2009 por Meulenhoff. Una baza a su favor o incluso decisiva fue la recepción espectacular de 2666 en los Estados Unidos en 2008, que fue motivo de varios artículos en la prensa holandesa y que de este modo creó ciertas expectativas. El gran eco de la novela en los Estados Unidos es el origen, asimismo, del eslogan "La sensación internacional por antonomasia" que, junto con una foto de Bolaño y una cita de Susan Sontag ("The most influential and admired novelist of his generation in the Spanish-speaking world"), constituye el conjunto paratextual que luce en la contracubierta de la edición holandesa (2666).

Todos los periódicos y semanarios importantes publicaron largas y positivas reseñas sobre 2666 y Bolaño se convirtió en the talk of the town. Pero si hubo un boom Bolaño en Holanda, este duró poco. A diferencia de Borges, García Márquez, Vargas Llosa e Isabel Allende, Roberto Bolaño apenas transcendió el estatus de one hit wonder: todos los demás libros del escritor chileno 
lanzados al mercado holandés después de 2666 (nuevas ediciones de las obras traducidas ya; nuevas traducciones) tuvieron una recepción efímera o rotundamente pobre, tanto en la prensa como entre los lectores. Incluso para 2666 la fiesta terminó pronto: la novela solo tuvo cuatro ediciones, todas en 2009. No es de sorprender, pues, que después de algunos años Meulenhoff, que se había comprometido a publicar toda la obra de Bolaño en Holanda, interrumpiera este proyecto. Después, el escritor chileno tuvo una segunda oportunidad en Lebowski, una editorial con una atinada sensibilidad publicitaria. Pero Lebowski tampoco fue capaz de encontrar un público para Bolaño. Por consiguiente, el autor que no pocos celebran como el nuevo Borges, carece ahora de casa editorial en Holanda. Es aguda la diferencia con el maestro argentino, cuya obra desde hace más de medio siglo sigue funcionando muy bien en De Bezige Bij.

\section{Desde Borges hasta García Márquez: César Aira}

Como hemos visto, no fue el marco de la narrativa latinoamericana el que contribuyó a catapultar a Bolaño a cierta fama en Holanda sino el éxito espectacular en los Estados Unidos de 2666. El dato no es azaroso sino que subraya que a lo largo de los años la literatura latinoamericana y sus valores más consagrados habían perdido en importancia y relevancia en su capacidad de marca y marco. Es inevitable, pues, la siguiente pregunta: ¿cómo se lanza a los nuevos valores del subcontinente en el nuevo milenio? ¿Cuáles son los contextos o marcos identitarios manejados por las editoriales en sus intentos de despertar la curiosidad y el interés de los lectores y, asimismo, el compromiso de importantes intermediarios como son los libreros y críticos? Por varias razones el caso de César Aira puede verse como representativo. La primera es que han sido varios los intentos de lanzar al autor en el mercado holandés. La segunda es que hay llamativas diferencias entre ellos. La tercera es que todos estos intentos han fracasado. Con todo, nos pueden dar una pista, ofreciéndonos una idea que está lejos de ser exhaustiva pero que es reveladora de la actual praxis editorial a la hora de introducir a nuevos valores literarios hispanoamericanos en el campo literario holandés.

La editorial De Geus lo tenía muy claro cuando en 2007 publicó la versión holandesa de Varamo, la novela con la que Aira debutó en los Países Bajos: lanzó a Aira como "el único verdadero alumno de Jorge Luis Borges - y el más talentoso", como reza la cita de un artículo publicado en el periódico alemán 
Frankfurter Allgemeine Zeitung que encabeza la cubierta del libro (De nachtelijke invallen). En la contracubierta figuran cinco citas de un variopinto abanico de fuentes: The Barcelona Review, Die Zeit, Inrockuptibles, Livres Hebdo y TagesAnzeiger. En ellas no se menciona a Borges, pero de forma implícita se refuerza el vínculo con el maestro argentino: en dos de ellas se destaca la nacionalidad del escritor (como se sabe, Aira es compatriota de Borges) y cuatro de ellas resaltan estrategias o rasgos literarios como la ironía, la ambigüedad, la ilusión, la originalidad, lo absurdo y la fantasía.

De nachtelijke invallen van ambtenaar Varamo, como reza el título de la versión holandesa, fue poco menos que negado en la prensa holandesa y tuvo tan pocas ventas que De Geus, una editorial que suele mantener una política de autor, dejó de invertir en Aira. En 2013 Meulenhoff le tomó relevo y publicó otra novela corta de Aira, De schimmen (Los fantasmas). Como en el caso de Varamo, hay una sola cita en la cubierta, que en este caso representa a Aira como un autor afín a Bolaño en base a una cita del autor chileno que no abarca más que una palabra: "Engancha”. En la contracubierta figuran citas de The New York Times, Der Spiegel y La Vanguardia, respectivamente, que no elaboran el presunto parentesco con Bolaño sino la originalidad de Aira: "Aira is one of the most provocative and idiosyncratic novelists working in Spanish today, and should not be missed” (The New York Times); "Aira hace una grotesca acrobacia literaria entre realismo y fantasía” (Der Spiegel); "Una y otra vez César Aira sorprende al lector con su rara genialidad" (La Vanguardia) ${ }^{3}$. En la minibiografía que figura en la contracubierta se vuelve a destacar la singularidad de Aira, esta vez en un marco más específico: "Se lo considera como uno de los autores más originales de América del Sur".

De schimmen no pasó desapercibida en la prensa. Los críticos no ponían en entredicho la originalidad de la novela. Tampoco discrepaban sobre la arduidad de la prosa de Aira aunque la apreciaban de distintos modos. En su reseña para el semanario De Groene que tiene como titular "Oda a lo inacabado" el joven escritor Christian Weijts formuló sus ideas encontradas sobre De schimmen de la siguiente manera: "Si entregara estas 99 páginas a mi editor este me diría: Estupendo borrador. Vamos a convertirlo en un libro fantástico”.

De schimmen fue un fracaso en ventas, pero no cabe duda de que el capital simbólico de Aira -acumulado gracias a las reseñas en la prensa holandesa, a la acogida en los Estados Unidos (donde en los años anteriores Aira se había convertido en un mini hype) y a los rumores sobre su candidatura para

3 Se trata de una versión muy libre del original: "César Aira es un escritor sorprendente cuyas obras de rara genialidad, se transmiten como un secreto entre una secta de entusiastas cada vez más amplia”. 
el premio Nobel- motivó a Meulenhoff a seguir con Aira. En 2016 la editorial publicó Hoe ik een non werd (Cómo me hice monja) en una edición atiborrada de citas paratextuales -De schimmen tiene cuatro, Hoe ik een non werd diez- que ensalzan la obra del escritor argentino desde distintas perspectivas, por distintos motivos y recurriendo a fuentes muy diversas. Parafraseando y resumiendo, rezan así: Aira sabe exactamente cómo seducir al lector (de Volkskrant), Aira es un autor excéntrico que engancha (Roberto Bolaño), Aira podrá ser el primer escritor argentino galardonado con el premio Nobel (Carlos Fuentes), Aira es uno de los autores más provocativos e idiosincrásicos en español (The New York Times), Por favor, dame otro libro de César Aira (Tubantia), César Aira sorprende al lector con su rara genialidad y tiene más y más aficionados (La Vanguardia), Aira hace una grotesca acrobacia literaria entre realismo y fantasía (Der Spiegel), Aira es un gran artesano que engancha (Die Zeit), Aira es uno de los autores más chocantes, excitantes y subversivos del mundo hispanohablante (El País), Aira escribe como un García Márquez que ha tomado drogas (Tages-Anzeiger). En su conjunto estos paratextos representan a Aira como un autor singular que engancha a sus lectores. Al mismo tiempo, camuflan la arduidad de su narrativa. Esta intrincada representación se condensa en la última cita, prominentemente presente en la contracubierta: "Aira escribe como un García Márquez que ha tomado drogas"4.

El lugar privilegiado de este último paratexto no deja de ser significativo: vincular a Aira al autor paradigmático de la nueva novela hispanoamericana no es evidente, pero aquí tiene todas las pintas de servir de último (y casi se diría desesperado) recurso manejado por Meulenhoff para "vender" a Aira a los lectores holandeses. No lo consiguió. Es más: Hoe ik een non werd ni siquiera fue señalada en la prensa.

\section{Conclusión}

En varios sentidos el caso de Aira es paradigmático. En primer lugar, cabe destacar que los paratextos de los libros de los nuevos valores del subcontinente que procuran encontrar un lugar en el campo literario holandés apenas refieren a la marca (y marco) "literatura latinoamericana" y sus variantes ("hispanoamericana", "suramericana") o a los autores asociados con la misma, algunos de los

\footnotetext{
4 Otra versión muy libre del original, que reza así: "Sein soeben auf Deutsch erschienener Roman "Die Mestizin” liest sich, als habe Gabriel García Márquez im LSD-Rausch die Hölle zum Paradies verklärt” (Blank).
} 
cuales -García Márquez, Vargas Llosa- en el curso de los años se convirtieron en marcas soberanas. En segundo lugar, Borges sigue siendo una firme referencia. En tercer lugar, se puede constatar que la marca Bolaño no tiene la presencia y potencia que tenían la literatura latinoamericana y sus valores más destacados en su momento. En cuarto lugar, se podrían interpretar el gran número y la llamativa diversidad de los paratextos en los libros de los nuevos valores como sintomáticos: no hay consenso sobre las marcas que podrían servir de marcos para los escritores latinoamericanos en busca de un público. Por un lado, es problemática esta ausencia. Por otro lado, empero, este estado de las cosas -que, si no me equivoco, no solo vale para Holanda- podría interpretarse como una emancipación, como una señal de que la literatura latinoamericana se está descontinentalizando, de que se está convirtiendo en literatura mundial.

\section{Bibliografía}

Aira, César. Hoe ik een non werd. Trad. Adri Boon. Meulenhoff, 2016.

--.. De schimmen. Trad. Adri Boon. Meulenhoff, 2013.

--.. De nachtelijke invallen van ambtenaar Varamo. Trad. Adri Boon. De Geus, 2007.

Allende, Isabel. Het huis met de geesten. Trad. Saskia Otter. Wereldbibliotheek, 1985.

“[a] unique design.” "Brand.” Business Dictionary. www.businessdictionary.com/definition/ brand.html, consultado 9 de julio 2018.

Blank, Gunter. “Wie Marquez auf LSD”. Sonntags Zeitung, 31 de octubre 2004, p. 53.

Bolaño, Roberto. 2666. Trad. Aline Glastra van Loon y Arie van der Wal. J.M. Meulenhoff, 2009.

---. De woeste zoekers. Trad. Aline Glastra van Loon. Meulenhoff, 2000.

Borges, Jorge Luis. De Aleph. Trad. A. Sillevis. De Bezige Bij, 1964.

De Vries, Corine. “Boekenweek?” de Volkskrant, 14 de marzo 1996, p. 15.

Esquivel, Laura. Rode rozen en tortilla's. Trad. Francine Mendelaar. Arena, 1991.

Kuipers, Willem. “Grootscheeps komt het Zuiden binnenzeilen”. de Volkskrant, 14 de febrero 1996, p. 4.

Steenmeijer, Maarten. "Los pasos perdidos: la primera presencia de la literatura hispanoamericana en Holanda”. América Latina y la literatura mundial: mercado editorial, redes globales y la invención de un continente. Ed. Gesine Müller y Dunia Gras, Iberoamericana/Vervuert, 2015, pp. 99-127.

Weijts, Christiaan. “Ode aan het onaffe”. De Groene Amsterdammer, 26 de noviembre 2013. 
